\title{
Depression, inflammation, and memory loss among Mexican Americans: analysis of the HABLE cohort
}

\author{
Leigh A. Johnson, ${ }^{1,2}$ Melissa Edwards, 1,2 Adriana Gamboa, 1,2 James Hall, 3,4 \\ Michelle Robinson ${ }^{5}$ and Sid E. O'Bryant ${ }^{1,2}$ \\ ${ }^{1}$ Center for Neuroscience Discovery, University of North Texas Health Science Center, Fort Worth, Texas, USA \\ ${ }^{2}$ Institute for Aging \& Alzheimer's Disease Research, University of North Texas Health Science Center, Fort Worth, Texas, USA \\ ${ }^{3}$ Department of Psychiatry, University of North Texas Health Science Center, Fort Worth, Texas, USA \\ ${ }^{4}$ Texas College of Osteopathic Medicine, Fort Worth, Texas, USA \\ ${ }^{5}$ Boehringer Ingelheim pharmaceuticals, Ridgefield, Connecticut, USA
}

ABSTRACT

Background: This study explored the combined impact of depression and inflammation on memory functioning among Mexican-American adults and elders.

Methods: Data were analyzed from 381 participants of the Health and Aging Brain study among Latino Elders (HABLE). Fasting serum samples were collected and assayed in duplicate using electrochemiluminesce on the SECTOR Imager 2400A from Meso Scale Discovery. Positive DepE (depression endophenotype) was codified as any score $>1$ on a five-point scale based on the GDS-30. Inflammation was determined by TNF $\alpha$ levels and categorized by tertiles (1st, 2nd, 3rd). WMS-III LMI and LMII as well as CERAD were utilized as measures of memory. ANOVAs examined group differences between positive DepE and inflammation tertiles with neuropsychological scale scores as outcome variables. Logistic regressions were used to examine level of inflammation and DepE positive status on the risk for MCI.

Results: Positive DepE as well as higher inflammation were both independently found to be associated with lower memory scores. Among DepE positive, those who were high in inflammation (3rd tertile) were found to perform significantly worse on WMS-III LM I $(\mathrm{F}=4.75, \mathrm{p}=0.003)$, WMS-III LM II $(\mathrm{F}=8.18$, $\mathrm{p}<0.001)$, and CERAD List Learning $(\mathrm{F}=17.37, \mathrm{p}<0.001)$ when compared to those low on inflammation (1st tertile). The combination of DepE positive and highest tertile of inflammation was associated with increased risk for $\mathrm{MCI}$ diagnosis $(\mathrm{OR}=6.06 ; 95 \% \mathrm{CI}=3.9-11.2, \mathrm{p}<0.001)$.

Conclusion: Presence of elevated inflammation and positive DepE scores increased risk for worse memory among Mexican-American older adults. Additionally, the combination of DepE and high inflammation was associated with increased risk for MCI diagnosis. This work suggests that depression and inflammation are independently associated with worse memory among Mexican-American adults and elders; however, the combination of both increases risk for poorer memory beyond either alone.

Key words: depression endophenotype (DepE), Mexican-Americans, mild cognitive impairment

Mexican-Americans often experience significant health disparities compared to non-Hispanic whites. As the fastest aging segment of the US population, Mexican-Americans face a significant health disparity when it comes to the development of neurodegenerative diseases. Mild cognitive impairment (MCI), considered as a prodromal category of neurodegenerative diseases, has been

Correspondence should be addressed to: Leigh A Johnson, Center for Neuroscience Discovery, University of North Texas Health Science Center, 3500 Camp Bowie Boulevard, Fort Worth, TX 76107, USA. Phone: 817-735-2965. Email: Leigh.Johnson@unthsc.edu. Received 3 Feb 2017; revision requested 26 Apr 2017; revised version received 16 May 2017; accepted 17 May 2017. First published online 20 June 2017. shown to be particularly high among MexicanAmericans (Dubious et al., 2007; Teixeria et al., 2012; O'Bryant et al., 2013a; 2013b; Alzheimer's Association, 2014). Risk factors for developing MCI among this population have been found to include diabetes, depression, and obesity (O'Bryant et al., 2013b; Johnson et al., 2015b; Downer et al., 2016). In our work, Mexican-Americans were found to have a higher prevalence of depressive symptoms (O'Bryant et al., 2007; O'Bryant et al., 2013b; O'Bryant et al., 2013c) and these symptoms were associated with worse cognitive functioning when compared to non-Hispanic whites. While some risk factors for neurodegenerative disease 
cannot be changed, depression has often been considered a modifiable risk factor for the development of MCI. Therefore more research is needed to understand the relationship between depression and memory loss in this underserved, yet burgeoning segment of the aging population.

Deconstructing the relationship between depression and memory may be hindered by our treatment of depression as a unitary construct. Our group has proposed that we must understand the influence of the impact of specific depressive symptoms on cognition. Our research team identified and validated a depressive endophenotype of cognitive impairment, called DepE (Johnson et al., 2013). Individuals with elevated DepE scores were at increased risk for MCI and Alzheimer's disease (AD) diagnoses, and demonstrated significantly poorer neurocognitive abilities at baseline and follow-up assessments. DepE was also tested in a cohort of cognitively normal cohort of adults from the Western Australian Memory Study, which found elevated DepE was associated with poorer cognitive performance among healthy cognitively normal individuals (Johnson et al., 2013).

Inflammatory processes have been proposed as a major component of the pathogenesis of both depression and dementia. Changes in serum acutephase proteins, chemokines, adhesion molecules, and pro-inflammatory cytokines have all been implicated in playing a role in the development of major depression. Increases in tumor necrosis factor- $\alpha$ (TNF $\alpha)$, interleukin 6 (IL6), and creactive protein have been consistently linked with increased depressive symptoms (Pucak and Kaplin, 2005; Simen et al., 2006; Losleben-Berthold and Himmerich, 2008 ; Miller et al., 2009; Trollor et al., 2012; Huang and Lin, 2015). TNF $\alpha$ concentrations have been found to be increased in depressed females as compared to healthy controls (Kahl et al., 2006). TNF $\alpha$ concentrations have also been linked to poorer cognition (Jefferson et al., 2011; Trollor et al., 2010) and the development of Alzheimer's (Buchhave et al., 2010; McCaulley and Grush, 2015). Windham et al. (2014) found a strong relationship between cognition and soluble TNF receptors 1 and 2 especially for AfricanAmericans.

The relationship between $\mathrm{TNF} \alpha$, cognition, and depression has not been extensively investigated. Matsushima et al. (2015) found no relationship between $\mathrm{TNF} \alpha$ and cognition and depression in a small sample of community-dwelling cognitively normal Japanese elderly. Lindqvist et al. (2013) in a study of Parkinson's patients using CSF inflammatory markers found no relationship between $\mathrm{TNF} \alpha$, depression, and MMSE scores. Although these studies indicate little relationship, the pathophysiology of $\mathrm{TNF} \alpha$ effecting cognition and mood suggests that a relationship may be found especially in populations where both depression and inflammatory diseases are prevalent, such as found for Mexican-Americans. In our work, inflammation was associated with diagnosis of MCI among Mexican-Americans (Edwards et al., 2015). The current study sought to examine the additive impact of elevated inflammation to DepE on memory abilities and risk for MCI diagnosis among community-dwelling MexicanAmerican adults and elders.

\section{Methods}

\section{Participants}

Data from 381 participants from the Health and Aging Brain among Latino Elders (HABLE) study were analyzed (Edwards et al., 2015; Johnson et al., 2015a; Szerlip et al., 2015). The HABLE study is an ongoing epidemiological study of cognitive aging among community-dwelling Mexican-American individuals. The HABLE study utilizes a communitybased participatory research (CBPR) approach, which is a research methodology that involves partnering communities with scientific groups to conduct studies of human disease. The generation of locations for targeted CBPR recruitment was determined through analysis of zip codes in Tarrant County with the highest population density of Hispanic individuals. This research was conducted under an IRB 2012-O83 approved protocol with each participant (and/or informants for cognitively impaired persons) providing writteninformed consent.

\section{Study design}

Each participant underwent an interview (i.e. medical history, medications, and health behaviors), detailed neuropsychological testing, blood draw, and medical examination (review of systems, Hachinski Ischemic Index scale, brief neurological screen). The neuropsychological battery consisted of tests of executive functioning: Trail Making Test, EXIT25, clock drawing (Sunderland et al., 1989; Royall et al., 1992; Strauss et al., 2006); language: FAS and Animal Naming (Strauss et al., 2006); visuospatial skills: CLOX2 (Sunderland et al., 1989); memory: (Wechsler Memory Scale - 3rd ed. (WMS-3) Logical Memory (Weschler, 1987) and Consortium for the Establishment of Registry for Alzheimer's Disease List Learning (Morris et al., 1989), and attention: WMS-3 Digit Span (Weschler, 1987). Testing was completed in English or Spanish depending on 
Table 1. Demographic characteristics

\begin{tabular}{|c|c|c|c|c|c|c|c|c|}
\hline & \multicolumn{3}{|c|}{ DEPE POSITIVE } & \multicolumn{4}{|c|}{ DEPE NEGATIVE } & \multirow[b]{2}{*}{ P-VALUE } \\
\hline & $\begin{array}{l}\text { TNF } \alpha \\
\text { TERTILE } 1 \\
\mathrm{~N}=67\end{array}$ & $\begin{array}{l}\text { TNF } \alpha \\
\text { TERTILE } 2 \\
\mathrm{~N}=53\end{array}$ & $\begin{array}{l}\text { TNF } \alpha \\
\text { TERTILE } 3 \\
\mathrm{~N}=62\end{array}$ & P-VALUE & $\begin{array}{l}\text { TNF } \alpha \\
\text { TERTILE } 1 \\
\text { N=57 }\end{array}$ & $\begin{array}{l}\text { TNF } \alpha \\
\text { TERTILE } 2 \\
\mathrm{~N}=74\end{array}$ & $\begin{array}{l}\text { TNF } \alpha \\
\text { TERTILE } 3 \\
\mathrm{~N}=79\end{array}$ & \\
\hline Age & $58.6(7.1)$ & $61.0(7.9)$ & $63.2(8.3)$ & $\begin{array}{l}1 \mathrm{vs} 2 \mathrm{NS} \\
1 \mathrm{vs} 3 \mathrm{p}=0.001 \\
2 \mathrm{vs} 3 \mathrm{NS}\end{array}$ & $59.0(6.6)$ & $60.1(9.2)$ & $62.7(7.9)$ & $\begin{array}{l}\text { 1vs } 2 \mathrm{NS} \\
1 \mathrm{vs} 3 \mathrm{p}=0.006 \\
2 \mathrm{vs} 3 \mathrm{NS}\end{array}$ \\
\hline Education & $7.3(3.6)$ & $6.1(3.3)$ & $6.6(3.8)$ & NS & $9.0(4.7)$ & $9.0(4.7)$ & $7.7(4.3)$ & NS \\
\hline $\begin{array}{l}\text { Gender } \\
\quad(\% \text { female })\end{array}$ & $90 \%$ & $80 \%$ & $71 \%$ & & $65 \%$ & $80 \%$ & $68 \%$ & \\
\hline
\end{tabular}

NS $=$ no significant.

the participant's preference. Here, analyses focused specifically on WMS-3 Logical Memory and CERAD List Learning scores. The DepE score was derived from the Geriatric Depression scale, which was also administered to all participants (Yesavage et al., 1982). Age and education corrected scale scores were utilized for analyses. The current team has generated normative references for each of these tests for English- and Spanish-speaking Mexican-Americans for diagnostic purposes (manuscripts in preparation). Cognitive diagnoses of MCI were assigned according to Mayo Clinic criteria (Peterson, 2003). Pre-MCI was defined as those who fell one standard deviation below the mean on CERAD List Recall but for whom were classified as cognitively normal. All diagnoses were determined through a consensus review panel.

\section{Blood collection and processing}

Blood collection and processing was conducted per international guidelines (O'Bryant et al., 2015). Briefly, fasting blood was collected as follows: (1) venipuncture using $21 \mathrm{~g}$ needle, (2) sample tubes collected in the following order - blood culture tube, coagulation tube, serum, heparin, plasma EDTA tube, (3a) serum tube will be allowed clot for 30 minutes at room temperature in a vertical position, (3b) plasma tubes gently inverted 5-10 times, (4) centrifuged with horizontal rotor for 10 minutes at $2,000 \times \mathrm{g}$ within one hour of collection, (5) $1.0-\mathrm{mL}$ aliquots of serum will be transferred into polypropylene (cryovial) tubes, (6) sample ID (Freezerworks ${ }^{\mathrm{TM}}$ barcode labels) affixed to each aliquot, and (7) samples will be placed into $-80{ }^{\circ} \mathrm{C}$ freezer within two hours of collection. Serum $\mathrm{TNF} \alpha$ was assayed in duplicate via a multi-plex biomarker assay platform using electrochemiluminesce (ECL) on the SECTOR Imager 2400A from Meso Scale Discovery (MSD; http://www.mesoscale.com) per our previously published methods (O'Bryant et al., 2013a). ECL measures have well-established properties of being more sensitive and requiring less sample volume than conventional ELISAs (Kuhle et al., 2010), the gold standard for most assays.

\section{Statistical analyses}

A positive score on DepE was codified as any score $>1$ on the five-point scale. TNF $\alpha$ levels were categorized by tertile scores $(1,2$, or 3$)$. Group differences by DepE positive $(>1)$ and $\mathrm{TNF} \alpha$ tertiles were examined by ANOVA with age and education corrected neuropsychological scale scores utilized as outcome variables. Additionally, ANCOVA analyses were conducted to verify the analyses with age, gender, and education used as covariates with the results remaining the same. Risk for MCI diagnosis by DepE positive and $\mathrm{TNF} \alpha$ third tertile was examined via logistic regression.

\section{Results}

Demographic characteristics of the sample by DepE scores and $\mathrm{TNF} \alpha$ tertiles are presented in Table 1 . As can be seen, in the DepE positive group those in the third tertile of TNF $\alpha$ were significantly older than those in the 1 st tertile. There were no significant differences in educational attainment. Among the DepE negative group, those in the 3rd tertile of $\mathrm{TNF} \alpha$ were significantly older than those in the 1 st tertile. There were no significant education discrepancies.

As can be seen from Table 2, those who are DepE positive score worse on memory measures than those who are DepE negative, which is consistent with our prior work (Johnson et al., 2013; Johnson et al., 2015a). When looking within DepE categories, increasing level of inflammation was associated with worse memory performance (see Table 2). 
Within the DepE positive group, those in the 3rd tertile of $\mathrm{TNF} \alpha$ performed worse on all measures. Specifically, those in the 3rd tertile of $\mathrm{TNF} \alpha$ scored significantly worse than those in the 1 st tertile on WMS-III Logical Memory $1(\mathrm{p}=0.003)$, Logical Memory $2(\mathrm{p}<0.001)$, and CERAD List Learning $(p<0.001)$. Those in the 2nd tertile of $\mathrm{TNF} \alpha$ performed significantly worse on WMS-III Logical Memory $1(\mathrm{p}=0.04)$, WMS-III LM $2(\mathrm{p}=0.01)$, and CERAD List Recall $(p=0.02)$. Those in the 3rd tertile of $\mathrm{TNF} \alpha$ also performed significantly worse than those in the 2nd tertile on the CERAD List Recall $(p=0.002)$. In the ANCOVA analyses, the results remained the same.

Within the DepE negative group, those participants in the 3rd tertile generally performed worse on memory tests than the other groups as well, although the findings were not as stark as among the DepE positive group. Those in the 3rd tertile of $\mathrm{TNF} \alpha$ performed significantly worse than the $1 \mathrm{st}$ tertile on the WMS-III LM2 $(\mathrm{p}=0.001)$. Those in the 3rd tertile of TNF $\alpha$ also performed significantly worse than those in the 2nd tertile on the WMS-III $\mathrm{LM} 2(\mathrm{p}=0.004)$ and CERAD $(\mathrm{p}=0.03)$.

In the logistic regression, being DepE positive $+\mathrm{TNF} \alpha 3$ rd tertile was associated with significant risk for MCI diagnosis $(\mathrm{OR}=6.06,95 \% \mathrm{CI}=3.9$ 11.2).

\section{Discussion}

This study examined the impact of inflammation and depression on memory among MexicanAmerican adults and elders. The results indicated that those with positive DepE scores and higher levels of inflammation performed poorer on all memory measures when compared to those with low inflammation. Furthermore, individuals with both positive DepE and placement in the highest inflammation tertiles were found to be at increased risk for MCI. The results suggest that inflammation may (1) play a key role in the relationship between depression and memory and/or (2) have an additive impact on memory decline when found in conjunction with depression.

Proinflammatory cytokines, such as $\mathrm{TNF} \alpha$, have been implicated in the pathophysiology of dementia and depression. Past research studies have found higher concentrations of $\mathrm{TNF} \alpha$ were found in depressed patients as compared to healthy controls (Dowlati et al., 2010) and inflammation has been consistently linked to cognitive dysfunction, $\mathrm{MCI}$, and AD in our prior work (O'Bryant et al., 2014; Edwards et al., 2015). However, studies examining the relationship between inflammatory markers, memory, and depression have been mixed. Several 
studies have found no relationship between $\mathrm{TNF} \alpha$ and cognition and depression (Matsushima et al., 2015). Himmerich et al. (2008) suggested these inconsistencies may be due to the subgroups of depression. It is possible that a specific subtype of depression is linked to inflammatory cytokines. This study is one of the first to examine the role of inflammation in a subgroup of depressed elders.

There are limitations of the current study. First, the current study utilized a cross-sectional design thus casual inferences may not be drawn for the results. Future research should examine the role of inflammation on the depression-cognition link utilizing a longitudinal design. Our group is currently collecting follow up data to examine this finding over time. Second, the current sample is insufficient to determine the relative contribution of inflammation or depression on cognition. Our group has received funding to increase the sample size of this cohort and we hope to be able to address this in future work. Next, this study examined only $\mathrm{TNF} \alpha$ levels. Future work should look at additional markers of inflammation to examine profiles that may further clarify this link. The focus on this study was on memory, however, future work should examine the impact of this relationship on other cognitive domains. Cognitive diagnoses were assigned via consensus review, and therefore not confirmed via PET scan or lumbar biomarker analyses. Thus, it is possible the MCI cases are not pre- $\mathrm{AD}$, as data on amyloid biomarkers was not available for this study. A significant advantage to this work; however, is that participants from this study were recruited from a community-based sample of elders rather than a clinic. Another significant advantage to this study is the sample size and the sample size within each subgroup is greater than 50. Last, this study utilized an underserved and understudied population. By 2050, the number of Hispanics in America over age 65 will nearly triple (Jacobsen et al., 2011), with Mexican-Americans making up over $65 \%$ of this group. Since advancing age is the greatest risk factor for developing cognitive impairment, Mexican-Americans will be disproportionately impacted by MCI/AD. Despite these facts, there are only a few aging research studies focusing on Mexican-Americans.

This study is the first to specifically examine the impact of inflammation on depression and memory among Mexican-American adults and elders. Utilizing the sub-group approach to classifying depression may help to elucidate the role inflammation plays in depression. Our past research has focused on identifying a subset of elders that experience depression related cognitive loss, and thus can accurately identify a selective group that may benefit from antidepressant treatment for prevention of memory loss and AD. These findings indicate that those individuals with elevated $\mathrm{TNF} \alpha$, are at great risk for memory loss, which may suggest a specific combination therapy approach to preventing and treating memory loss among this specific subgroup of Mexican-American older adults. Further work is warranted to continue to push forward this notion of precision medicine that is tailored to specific ethnic groups, which is akin to ongoing efforts in the cancer, diabetes, and cardiovascular disease spaces. While this work focused specifically on memory loss, the current team is expanding the analyses to examine the impact of depression and inflammation on other cognitive domains, including executive functioning, language, attention/processing, and visuospatial abilities

\section{Conflict of interest}

None.

\section{Authors' contributions}

Conceived and designed the experiments: LAJ, AG, ME, JH, MR, SO. Performed the experiments: LAJ, AG, ME, JH, MR, SO. Analyzed the data: LAJ, AG, ME, MR, JH, SO. Contributed reagents/materials/analysis tools: LAJ, AG, ME, MR, JH, SO. Wrote the manuscript: LAJ, AG, MR, $\mathrm{ME}, \mathrm{JH}$, SO. Other: ICMJE criteria for authorship read and met: LAJ, AG, ME, MR, JH, SO. Agree with manuscript results and conclusions: LAJ, AG, ME, MR, JH, SO.

\section{Acknowledgments}

Research reported in this publication was supported by the National Institutes of Health under Award Number R01AG039389. The content is solely the responsibility of the authors and does not necessarily represent the official views of the National Institutes of Health. This research was also funded with grant from Alzheimer's Association AARG-16-442652, The Pfeiffer foundation, and the National Academy of Neuropsychology. The authors would additionally like to thank the Health and Aging Brain Study and research team.

\section{Ethics approval and consent to participate}

This research was conducted under an IRB 2012O83 approved protocol with each participant (and/or informants for cognitively impaired persons) providing written informed consent. 


\section{List of abbreviations}

CBPR, community-based participatory research

CERAD, Consortium for the Establishment of Registry for Alzheimer's Disease

DepE, depression endophenotype

ECL, electrochemiluminesce

HABLE, Health and Aging Brain study among Latino Elders

IL6, interleukin 6

LM, logical memory

MCI, mild cognitive impairment

$\mathrm{TNF} \alpha$, tumor necrosis factor- $\alpha$

WMS-III, Wechsler Memory Scale - 3rd ed.

\section{References}

Alzheimer's Association (2014). Alzheimer's disease facts and figures. Alzheimer's $\mathcal{E}$ Dementia, 10, e47-e92.

Buchhave, P., Zetterberg, H., Blennow, K., Minthon, L., Janciauskiene, S. and Hansson, O. (2010). Soluble TNF receptors are associated with $\mathrm{A} \beta$ metabolism and conversion to dementia in subjects with mild cognitive impairment. Neurobiology Aging, 11, 1877-1884.

Dowlati, Y. et al. (2010). Meta-analysis of cytokines in major depression. Biological Psychiatry, 67, 446-457.

Downer, B., Vickers, B. N., Al Snih, S., Raji, M. and Markides, K. S. (2016). Effects of comorbid depression and diabetes on cognitive decline among older Mexican Americans. Fournal of the American Geriatrics Society, 64, 109-117. doi:10.1111/jgs.13883.

Dubois, B. et al. (2007). Research criteria for the diagnosis of alzheimer's disease: revising the NINCDS-ADRDA criteria. Lancet Neurology, 6, 734-746.

Edwards, M., Balldin, V. H., Hall, J. and O'Bryant, S. E. (2015). Molecular markers of neuropsychological functioning and Alzheimer's disease. Alzheimer's $\mathcal{E}$ Dementia: Diagnosis, Assessment and Disease Monitoring, 1, 61-66.

Himmerich, H. et al. (2008). Depression, comorbidities and the TNF- $\alpha$ system. European Psychiatry, 23, 421-429.

Huang, T. L. and Lin, C. C. (2015). Advances in biomarkers of major depressive disorder. Advances in Clinical Chemistry, 68, 177-204.

Jacobsen, L. A., Mather, M. and Lee, M. (2011). America's aging population. Population Bulletin 66, no. 1. http://www.prb.org/pdf11/aging-in-america.pdf.

Jefferson, A. L. et al. (2011). Inflammatory markers and neuropsychological functioning: the Framingham Heart Study. Neuroepidemiology, 37, 21-30.

Johnson, L. A. et al. (2015a). A depressive endophenotype of poorer cognition among cognitively healthy community-dwelling adults: results from the western australia memory study. International fournal of Geriatric Psychiatry, 30, 881-886.

Johnson, L. A. et al. (2015b). Comorbid depression and diabetes as a risk for mild cognitive impairment and alzheimer's disease in elderly Mexican Americans. Fournal of Alzheimer's Disease, 47, 129-136.

Johnson, L. A., Hall, J. R. and O'Bryant, S. E. (2013). A depressive endophenotype of mild cognitive impairment and alzheimer's disease. Plos One, 8, e68848.

Kahl, K. G. et al. (2006). Cortisol, the cortisol-dehydroepiandrosterone ratio, and pro-inflammatory cytokines in patients with current major depressive disorder comorbid with borderline personality disorder. Biological Psychiatry, 59, 667-671.

Kuhle, J. et al. (2010). A highly sensitive electrochemiluminescence immunoassay for the neurofilament heavy chain protein. fournal of Neuroimmunology, 220, 114-119.

Lindqvist, D. et al. (2013). Cerebrospinal fluid inflammatory markers in Parkinson's disease-associations with depression, fatigue, and cognitive impairment. Brain Behavior and Immunity, 33,183-189.

Losleben-Berthold, M. and Himmerich, H. (2008). The TNF-alpha system: functional aspects in depression, narcolepsy and psychopharmacology. Current Neuropharmacology, 6, 193-202.

Matsushima, J. et al. (2015). Association of inflammatory biomarkers with depressive symptoms and cognitive decline in a community-dwelling healthy older sample: A 3-year follow-up study. Fournal of Affective Disorders, 173, 9-14.

McCaulley, M. E. and Grush, K. A. (2015). Alzheimer's disease: Exploring the role of inflammation and implications for treatment. International fournal of Alzheimer's Disease, Article ID 515248, 10 pages, doi: $10.1155 / 2015 / 515248$.

Miller, A. H., Maletic, V. and Raison, C. L. (2009). Inflammation and its discontents: the role of cytokines in the pathophysiology of major depression. Biological Psychiatry, 65, 732-741.

Morris, J. C. et al. (1989). The consortium to establish a registry for Alzheimer's disease (CERAD). Part I. clinical and neuropsychological assessment of Alzheimer's disease. Neurology, 39, 1159-1165.

O'Bryant, S. E. et al. (2013a). Molecular neuropsychology: creation of test-specific blood biomarker algorithms. Dementia \& Geriatric Cognitive Disorders, 37, 45-57.

O'Bryant, S. E. et al. (2013b). Characterization of Mexican Americans with mild cognitive impairment and Alzheimer's disease. Fournal of Alzheimer's Disease, 33, 373-379. PMID: 22976076.

O'Bryant, S. E. et al. (2013c). Risk factors for mild cognitive impairment among Mexican Americans. Alzheimer's E Dementia, 9, 622-631.

O'Bryant, S. E. et al. (2014). Validation of a serum screen for Alzheimer's disease across assay platforms, species and tissues. Fournal of Alzheimer's Disease, 42, 1325-1335.

O'Bryant, S. E. et al. (2015). Guidelines for the standardization for preanalytic variables for blood-based biomarker studies in Alzheimer's disease research. Alzheimer's E Dementia, 11, 549-560.

O'Bryant, S. E., Humphreys, J. D., Sutker, P. B. and Schiffer, R. B. (2007). Presentation of Mexican American patients to a memory disorder clinic. Fournal of Psychopathology and Behavioral Assessment, 29, 137-140. 
Petersen, R. C. (2003). Mild Cognitive Impairment: Aging to Alzheimer's Disease. New York: Oxford University Press.

Pucak, M. L. and Kaplin, A. I. (2005). Unkind cytokines, current evidence for the potential role of cytokines in immune-mediated depression. International Review of Psychiatry, 17, 477-483.

Royall, D., Mahurin, R. K. and Gray, K. (1992). Bedside assessment of executive cognitive impairment: The executive interview (EXIT). Fournal of American Geriatric Society, 40, 1221-1226.

Simen, B. B., Duman, C. H., Simen, A. A. and Duman, R. S. (2006). TNF $\alpha$ signaling in depression and anxiety: Behavioral consequences of individual receptor targeting. Biological Psychiatry, 59, 775-785.

Strauss, E., Sherman, E. M. S. and Spreen, O. (2006). $A$ Compendium of Neuropsycholgoical Tests: Administration, Norms, and Commentary, 3rd ed. Oxford: Oxford University Press.

Sunderland, T. et al. (1989). Clock drawing in Alzheimer's disease. A novel measure of dementia severity. Fournal of the American Geriatric Society, 37, 725-729.

Szerlip, H. M., Edwards, M. L., Williams, B. J., Johnson, L. A., Vintimilla, R. M. and O'Bryant, S. E. (2015). Association of cognitive impairment with chronic kidney disease in Mexican Americans. Fournal of the American Geriatric Society, 63, 2023-2028.

Teixeria, C. V. L., Gobbi, L. T. B., Corazza, D. I., Stella, F., Costa, J. L. R. and Gobbi, S. (2012).

Non-pharmacological interventions on cognitive function in older people with mild cognitive impairment (MCI). Archives of Gerontology and Geriatrics, 54, 175-180.

Trollor, J. N. et al. (2010). Systemic inflammation is associated with MCI and its subtypes: the Sydney memory and aging study. Dementia and Geriatric Cognitive Disorders, 30, 569-578.

Trollor, J. N. et al. (2012). The association between systemic inflammation and cognitive performance in the elderly: the Sydney Memory and Ageing Study. Age (Dordr.), 34, 1295-1308.

Weschler, D. (1987). Weschler Memory Scale-Revised. San Antonio, TX: Psychological Corporation.

Windham, B.G. et al. (2014). Associations between inflammation and cognitive function in african americans and european americans. The American Geriatrics Society, 62, 2303-2310.

Yesavage, J. A. et al. (1982). Development and validation of a geriatric depression screening scale: a preliminary report. Fournal of Psychiatric Research, 17, 37-49. 